\title{
Salivary anti-nuclear antibody (ANA) mirrors serum ANA in systemic lupus erythematosus
}

Ting Zhang ${ }^{1,2}$, Yong Du', Qingqing Wu ${ }^{1}$, Hao Li ${ }^{1}$, Thao Nguyen ${ }^{1}$, Gabriel Gidley ${ }^{1}$, Valeria Duran ${ }^{1}$, Daniel Goldman ${ }^{3}$, Michelle Petri ${ }^{3}$ and Chandra Mohan ${ }^{1 *}$ (1)

\begin{abstract}
Objectives: To assay salivary anti-nuclear antibody (ANA) and its isotypes in patients with systemic lupus erythematosus (SLE) and to investigate relevant clinical associations.

Methods: Saliva samples were collected from SLE patients and assayed for salivary ANA using immunofluorescence (IF). Isotypes of salivary ANA, including IgG-ANA, IgA-ANA, and IgM-ANA, were quantified using enzyme-linked immunosorbent assay. The correlations between clinical parameters and levels of salivary ANA and isotypes were evaluated.

Results: Salivary ANA IF intensities were significantly higher in SLE patients than in healthy controls, irrespective of SLE patient disease activity, and strongly correlated with serum ANA titers. Salivary ANA was detected in $67.14 \%$ of SLE patients and $10.00 \%$ of healthy controls $(p<0.001)$. Among ANA-positive samples, $80.85 \%$ exhibited a nuclear ANA pattern, and $42.55 \%$ exhibited a cytoplasmic ANA pattern. Salivary IgG-ANA, IgA-ANA, and IgM-ANA levels, as assayed by ELISA, were significantly increased in both active and less active SLE patients compared with healthy controls, and levels of each isotype were significantly correlated with serum ANA titer. Salivary IgM-ANA levels correlated with the physician global assessment (PGA), SLE disease activity index (SLEDAI), and negatively with serum C3 and C4. Salivary IgG-ANA also correlated with erythrocyte sedimentation rate (ESR), SLEDAl, and negatively with serum C3.
\end{abstract}

Conclusion: Salivary ANA levels correlate with serum ANA titer, and salivary IgM-ANA and IgG-ANA correlate variably with PGA, SLEDAI, ESR and complement levels. These findings underscore the potential of using salivary ANA and ANA isotypes as surrogates for serum ANA, particularly for future point-of-care applications since saliva is easier to obtain than blood.

Keywords: SLE, Saliva, ANA, Isotype

\section{Introduction}

Anti-nuclear antibody (ANA), consisting of diverse autoantibodies targeting nuclear and cytoplasmic cell components, is the serological hallmark of systemic lupus erythematosus (SLE) and is present in 95-99\% of SLE patients [1-4]. Given the rarity of ANA-negative SLE patients, detection of ANA is critical to SLE diagnosis [5]. In 2019, the European League Against Rheumatism

*Correspondence: cmohan@central.uh.edu

${ }^{1}$ Biomedical Engineering Department, University of Houston, 3517 Cullen Blvd, Room 2027, TX, Houston, USA

Full list of author information is available at the end of the article and the American College of Rheumatology defined the minimum criterion for SLE diagnosis as an ANA titer of $\geq 1: 80$ or at least one equivalent positive test, reflecting use of ANA as a sensitive SLE screening test [2]. ANA has also been used as an entry criterion for clinical trials of new therapeutic agents for SLE [1]. Moreover, autoantibodies play essential roles in SLE pathogenesis and have been implicated in immune complex formation and subsequent type I interferon production $[1,6,7]$. Serum ANA isotypes, including immunoglobulin G (IgG)-ANA, IgM-ANA, and IgA-ANA, have been identified in SLE patients and may have distinct roles in SLE pathogenesis $[8,9]$. However, the clinical associations and potential 
implications of ANA isotypes in SLE pathogenesis are unclear.

The current standard for ANA testing is the immunofluorescence (IF) assay using human epithelial type-2 (HEp-2) cells, which contain various autoantigens, as substrate [4, 10]. Enzyme-linked immunosorbent assay (ELISA) has also been used for quantitative ANA measurement [11]. Although ANA is most commonly detected in serum, it is also present in various SLE patient body fluids, including pleural effusion [12], cerebrospinal fluid [13], synovial fluid [14], and urine [15]. However, ANA has not been assayed in saliva of SLE patients.

Saliva, a readily accessible specimen, has emerged as a tool for non-invasive assessment of patient health [16]. Saliva comprises several components with multiple functions and diagnostic values [17], including many plasma proteins [18]. Thus, saliva may be a useful tool for diagnosing systemic diseases. Aside from oral diseases [18], saliva biomarkers have been used to evaluate diabetes mellitus [19], acute myocardial infarction [20], and lung cancer [21]. In patients with primary Sjögren's syndrome (pSS), an autoimmune disease with direct involvement of salivary glands, anti-SSA/B antibodies, which are included in the ANA spectrum, have been measured in saliva and assessed for diagnosis [22-24]. Given the ongoing efforts to develop point-of-care monitoring devices for rapid SLE diagnosis or home-based monitoring, this pilot study aimed to ascertain whether saliva is a viable biofluid for ANA detection and to investigate salivary ANA isotypes and their clinical correlations in SLE.

\section{Methods \\ Study population}

Saliva samples $(n=70)$ were obtained from SLE patients from the Division of Rheumatology, Johns Hopkins University (JHU) School of Medicine (Baltimore, MD, USA). Saliva samples of healthy individuals $(n=10)$ were obtained from BioIVT (New York, NY, USA) or the University of Houston (Houston, TX, USA). Informed consent was obtained from each participant, and the study was approved by the Institutional Review Boards of the JHU School of Medicine and the University of Houston. All SLE patients met the 2012 Systemic Lupus International Collaborating Clinics SLE classification criteria [5]. The following parameters were recorded: SLE disease activity index (SLEDAI), renal domains of SLEDAI (rSLEDAI), physician global assessment (PGA), complete blood count, serum creatinine, complement $\mathrm{C} 3$ and $\mathrm{C} 4$, serum ANA, and serum anti-double stranded (ds)DNA. SLE patients were categorized into two groups based on SLEDAI: (1) less active patients with SLEDAI $\leq 4$ and clinical SLEDAI (omitting dsDNA and complement) $\leq$ 2 and (2) active SLE with SLEDAI $\geq 5$ or rSLEDAI $\geq 4$.
Patients were not involved in the study design and conduct of this research.

\section{Sample collection}

Whole saliva samples were collected between $7 \mathrm{AM}$ and $8 \mathrm{AM}$ using the Salivette ${ }^{\circledR}$ hygienic saliva collection device (Sarstedt, Nümbrecht, Germany) according to manufacturer instructions. Before sample collection, patients and healthy volunteers refrained from food or drink for $\geq 30 \mathrm{~min}$, removed lipstick/balm, and rinsed their mouths without brushing or flossing teeth. The cotton swab was placed directly into the mouth, gently chewed and rolled around in the mouth for $3 \mathrm{~min}$, and spat back into the tube. The capped tube was centrifuged at $1000 \times g$ for $2 \mathrm{~min}$ to yield a clear saliva sample that was collected, aliquoted, and stored at $-80^{\circ} \mathrm{C}$. The saliva volume yield ranged between 0.8 and $1.4 \mathrm{ml}$ per individual.

\section{Indirect immunofluorescence}

Saliva ANA was assayed by immunofluorescence (IF) using a commercial ANA testing kit (Catalog number: ANK120, MBL Bion, Des Plaines, IL, USA). Briefly, saliva samples were added undiluted to wells pre-coated with HEp-2 cells, incubated in a moist chamber at room temperature for $30 \mathrm{~min}$, and washed with phosphate-buffered saline (PBS). Fluorescein isothiocyanate (FITC)-conjugated goat anti-human immunoglobulin, which detects the total immunoglobulin, was added to each well, and the slide was incubated for $30 \mathrm{~min}$ in the dark. After a PBS wash, mounting medium and coverslip were added, and the slide was examined with a confocal microscope (Nikon, Tokyo, Japan). ANA isotypes were assayed following a similar procedure using DyLight 650 conjugated goat anti-human IgG Fc antibody (1:500, Catalog Number SA5-10137, Thermo Fisher Scientific, Rockford, IL, USA), FITC-conjugated goat anti-human IgM (heavy chain) antibody (1:1000, Catalog Number A18842, Thermo Fisher Scientific), and tetramethylrhodamine isothiocyanate (TRITC)-conjugated goat anti-human IgA antibody (1:1000, Catalog Number A18786, Thermo Fisher Scientific). The ANA patterns were categorized according to the international consensus on standardized nomenclature of ANA HEp-2 cell patterns [25]. The IF staining intensities were graded independently by four trained observers (TZ, YD, QW, and $\mathrm{HL}$ ) using a $0-4$ scale based on comparison with a standard reference gallery (https://wwwn.cdc.gov/Nchs/Nhanes/1999-2000/ SSANA_A.htm). The average score for each sample was calculated and recorded as the "observer score" (OS). The IF intensity of each sample was quantified using ImageJ $(\mathrm{NIH})$ and recorded as the "ImageJ score" (IS). 
Elisa

To assay salivary ANA isotypes, ELISAs were performed using commercially available kits (INOVA Diagnostics, Inc., San Diego, CA, USA) containing microplates pre-coated with HEp-2 substrates. Saliva samples were diluted 1:2 in sample diluent. IgG-ANA was detected with the anti-IgG antibody provided in the kit. IgMANA and IgA-ANA were detected with horseradish peroxidase (HRP)-conjugated goat anti-human IgM antibody (1:20000, Catalog number 109-035-043, Jackson ImmunoResearch Laboratories, West Grove, PA, USA) and anti-human IgA antibody (1:20000, Catalog number 109-035-011, Jackson ImmunoResearch Laboratories), respectively. Standard curves for the isotypes were established with human IgG, IgA, and IgM ELISA kits (Abcam, Cambridge, MA, USA). Optical density at $450 \mathrm{~nm}$ was measured with a microplate reader (ELX808, BioTek Instruments, Winooski, VT, USA) and used to calculate protein concentrations.

\section{Statistical analyses}

Data were analyzed using GraphPad Prism 7 and R. The Mann-Whitney $U$ test was applied for comparisons between two groups, and chi-square test or Fisher's exact test was used to compare percentages. The nonparametric Spearman correlation was performed for correlation analysis. The receiver operating characteristic (ROC) curve was used to determine optimal cut-off values. A two-tail $p$-value of less than 0.05 was considered significant.

\section{Results}

\section{SLE patients exhibit higher salivary ANA IF intensities} than healthy controls

Saliva samples from 70 SLE patients and 10 healthy controls were evaluated for IF intensity (Supplementary Table S1). The OS and IS values used to quantify the IF intensities of salivary ANA were highly correlated $(R=0.77, p<0.0001)$ (Fig. 1A). The salivary ANA IF intensities were significantly higher in SLE patients, irrespective of disease activity, than in healthy controls (all $p<0.01$ ) (Fig. 1B, C). The OS cutoff value used to differentiate samples from SLE patients and healthy individuals was determined by ROC analysis (Fig. 1D). With a salivary ANA OS cutoff value of 0.75 , the corresponding salivary ANA positivity rate in healthy volunteers and

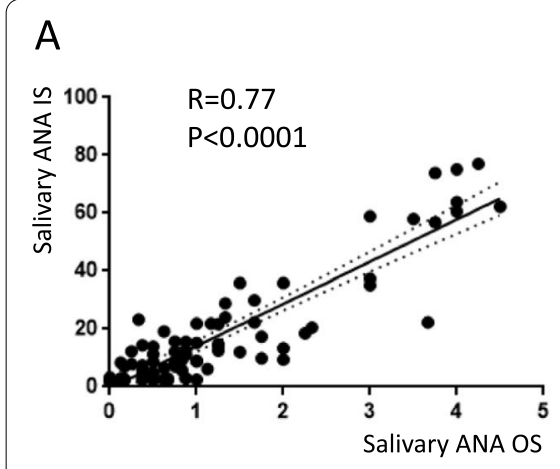

D

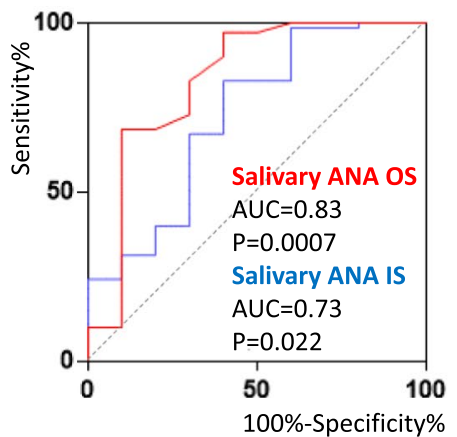

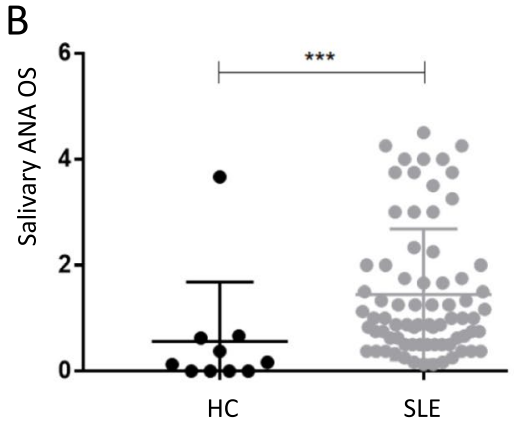

$\mathrm{E}$

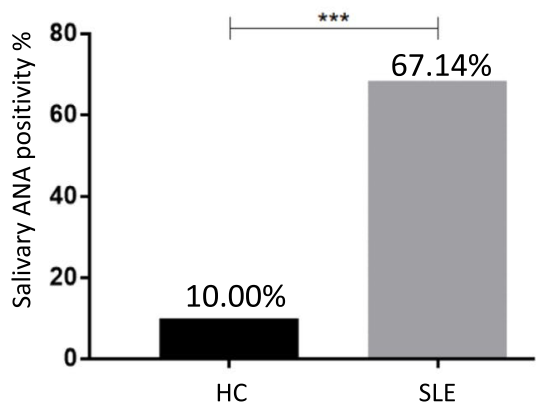

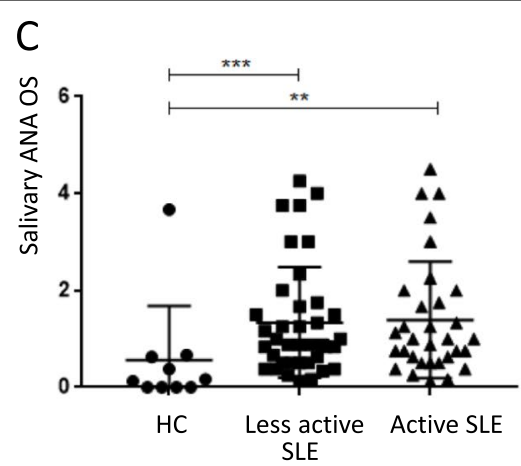

$\mathrm{F}$

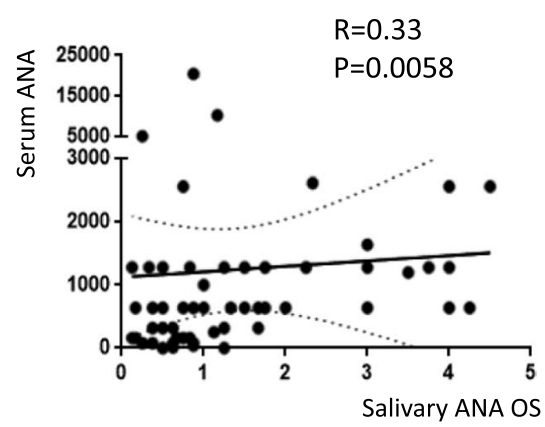

Fig. 1 Salivary ANA positivity and IF intensity were significantly higher in SLE patients than in HC. A Correlation of salivary ANA observer score (OS) and ImageJ score (IS) for ANA IF intensity. Salivary ANA OS represents the average observer score of four observers. B ANA IF intensity was significantly higher in SLE patients $(n=70)$ than in HC $(n=10)$, C irrespective of the disease status of SLE patients (less active SLE, $n=38$; active SLE, $n=32$ ). D ROC analysis illustrating the ability of saliva ANA IF intensities to differentiate SLE patients from HC. E ANA positivity of SLE and HC defined by the cutoff value of $O S \geq 0.75$. F Saliva ANA OS was significantly correlated with serum ANA titer. ${ }^{*} P<0.05,{ }^{* *} P<0.01,{ }^{* * *} P<0.001$. ANA, anti-nuclear antibodies; IF, immunofluorescence; HC, healthy controls 
patients with SLE was $10.00 \%$ and $67.14 \%$, respectively $(p=0.0009)$ (Fig. 1E). Moreover, the salivary ANA IF intensities of SLE patients were significantly correlated with serum ANA titers (Fig. 1F).

\section{Salivary ANA patterns}

Among the 47 ANA-positive SLE patients (salivary ANA OS $\geq 0.75), 38(80.85 \%)$ exhibited a nuclear ANA pattern, and $20(42.55 \%)$ exhibited a cytoplasmic staining pattern, with some individuals exhibiting more than one ANA staining pattern. Some samples demonstrated typical ANA sub-patterns, such as nuclear-homogeneous, nuclear-speckled, nuclear-nucleolar, and cytoplasmic speckled (Fig. 2A-D). Because further dilutions were not performed, the sub-patterns of some samples with strong IF were not confirmed, particularly samples that exhibited seemingly homogeneous nucleoplasmic staining. One healthy individual had positive salivary ANA with a nuclear pattern (not shown). ANA patterns did not correlate with disease activity as determined by SLEDAI (data not shown).

\section{ELISA quantification of salivary ANA isotypes}

The concentration of each salivary ANA isotype was measured by ELISA and found to be significantly higher in SLE patients than in healthy controls. Salivary ANA isotypes were significantly elevated in both active and less active SLE compared to healthy controls. ROC analysis indicated that each salivary ANA isotype significantly discriminated SLE patients from healthy individuals (Fig. 3). Whereas IgM ANA had the highest ROC AUC value (0.77), IgA ANA exhibited the highest sensitivity for SLE (at 0.80). Each ANA isotype was defined as either negative or positive based on the cutoff value resulting from ROC analysis, and combinations of ANA isotypes in discriminating SLE from healthy controls were also evaluated. However, all pairwise combinations or triple combination of ANA isotypes did not improve the discriminatory power of salivary autoantibodies (Supplementary Fig. S1).

\section{Correlation of salivary ANA and ANA isotypes with clinical features}

The salivary ANA OS and IS (which correlate with each other, Fig. 1A), as well as the concentrations of each ELISA-assayed salivary ANA isotype, were significantly correlated with serum ANA titers (Fig. 4). The salivary concentration of each isotype was significantly correlated with serum anti-dsDNA antibody, but a similar correlation was not observed for salivary ANA OS or IS. Salivary IgM-ANA concentration also correlated with PGA, SLEDAI, and serum C3 and C4, but did not correlate
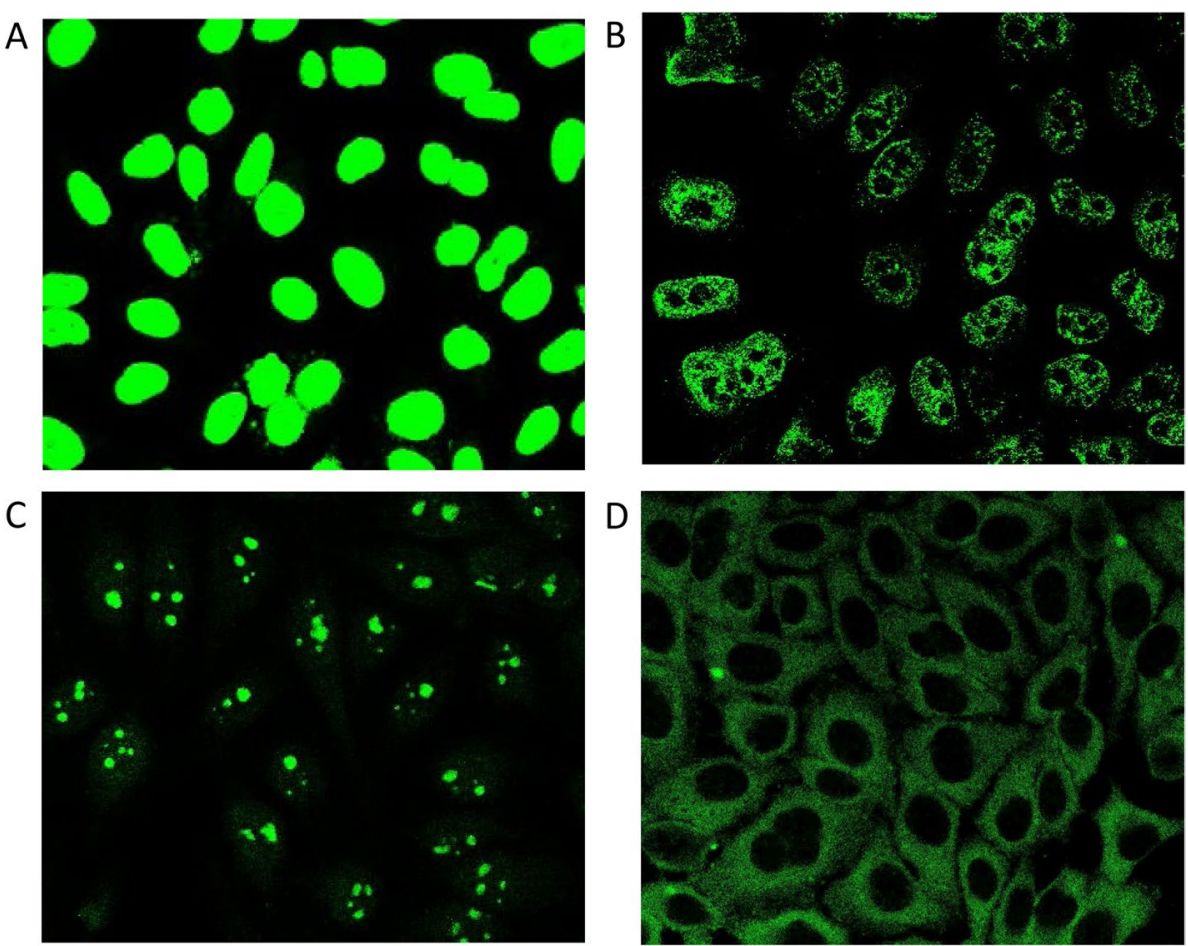

Fig. 2 Examples of diverse salivary ANA patterns in 47 saliva-ANA-positive SLE patients using immunofluorescence. A Nuclear-homogeneous. B Nuclear-speckled. C Nuclear-nucleolar. D Cytoplasmic-speckled 


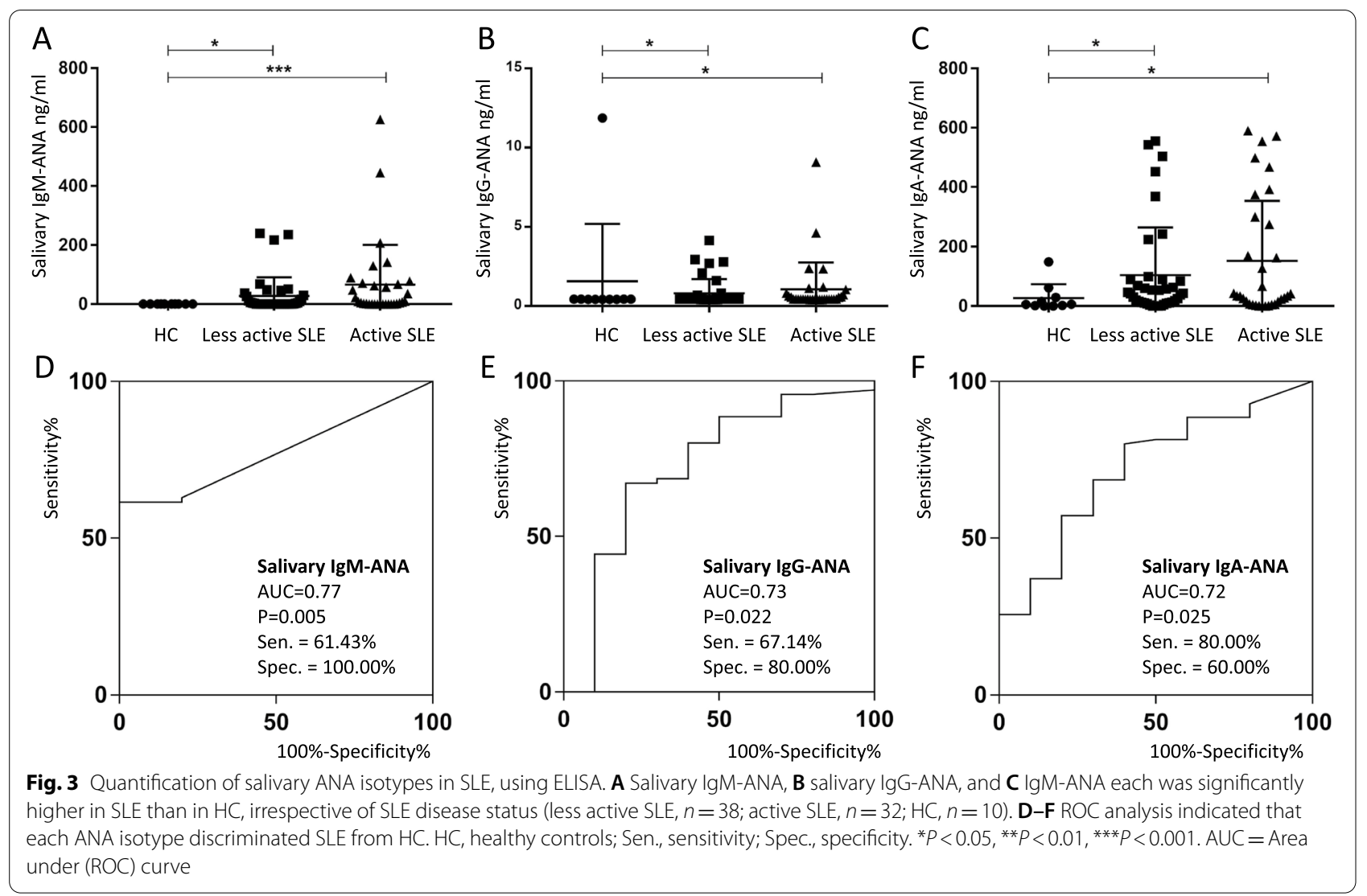

with erythrocyte sedimentation rate (ESR). Additionally, salivary IgG-ANA concentration correlated with ESR, SLEDAI, and serum C3, and IgA-ANA concentration correlated with ESR (Fig. 4). Although these correlations were significant, they were modest, with correlation coefficients ranging from 0.42 to -0.35 (Supplementary Table S2).

The anti-nuclear staining patterns by ANA of different isotypes in saliva of SLE patients were next examined using IF. Salivary ANA isotypes, including IgG-ANA, IgA-ANA, and IgM-ANA, from saliva of 3 SLE patients and 1 ANA+ve healthy control stained nuclei with different patterns, with some overlap between the staining patterns observed between the isotypes, as determined by IF. These limited IF studies did not reveal any evidence to suggest that salivary ANAs of different isotypes might be targeting different cellular epitopes (Fig. 5).

\section{Discussion}

Saliva mainly originates from three paired major salivary glands and numerous minor salivary glands that generate $0.75-1.5 \mathrm{~L}$ of this exocrine secretion per day [18]. Saliva also contains fluids derived from oropharyngeal mucosae and gingival crevices. Plasma compounds can enter saliva by ultrafiltration through gap junctions, transudation, or selective transport through cellular membranes [16], and $20-57.1 \%$ of saliva proteins are also found in the plasma [18, 26-28]. Thus, saliva proteins can provide insight into systemic conditions and may be useful for disease monitoring or early diagnosis.

Saliva proteins perform multiple functions, including lubrication, digestion, and protection [29]. As many as 5500 proteins have been identified in saliva by proteomics $[26,30]$. Immunoglobulins, including $\operatorname{IgA}, \operatorname{IgG}$, and IgM, account for $5-15 \%$ of total salivary proteins $[16$, 17]. However, variable salivary concentrations have been reported for each isotype [23,31-33]. Although IgG is the dominant immunoglobulin in serum, IgA comprises $>85 \%$ of salivary immunoglobulins [16, 17]. Salivary IgA is predominantly produced as secretory $\operatorname{IgA}(\operatorname{sigA})$ as a dimeric form by B lymphocytes located near the salivary glands [34]. Monomeric IgA derived from the serum may also be detected in whole saliva [35]. Pentameric IgM is secreted by the same mechanism as IIgA, and salivary IgG mainly derives from serum by passive diffusion [16, 17, 34]. Whether salivary ANA isotypes are generated from the same origins as immunoglobulin isotypes in saliva is unknown. Nevertheless, the elevated salivary ANA isotypes observed in SLE patients are unlikely to 


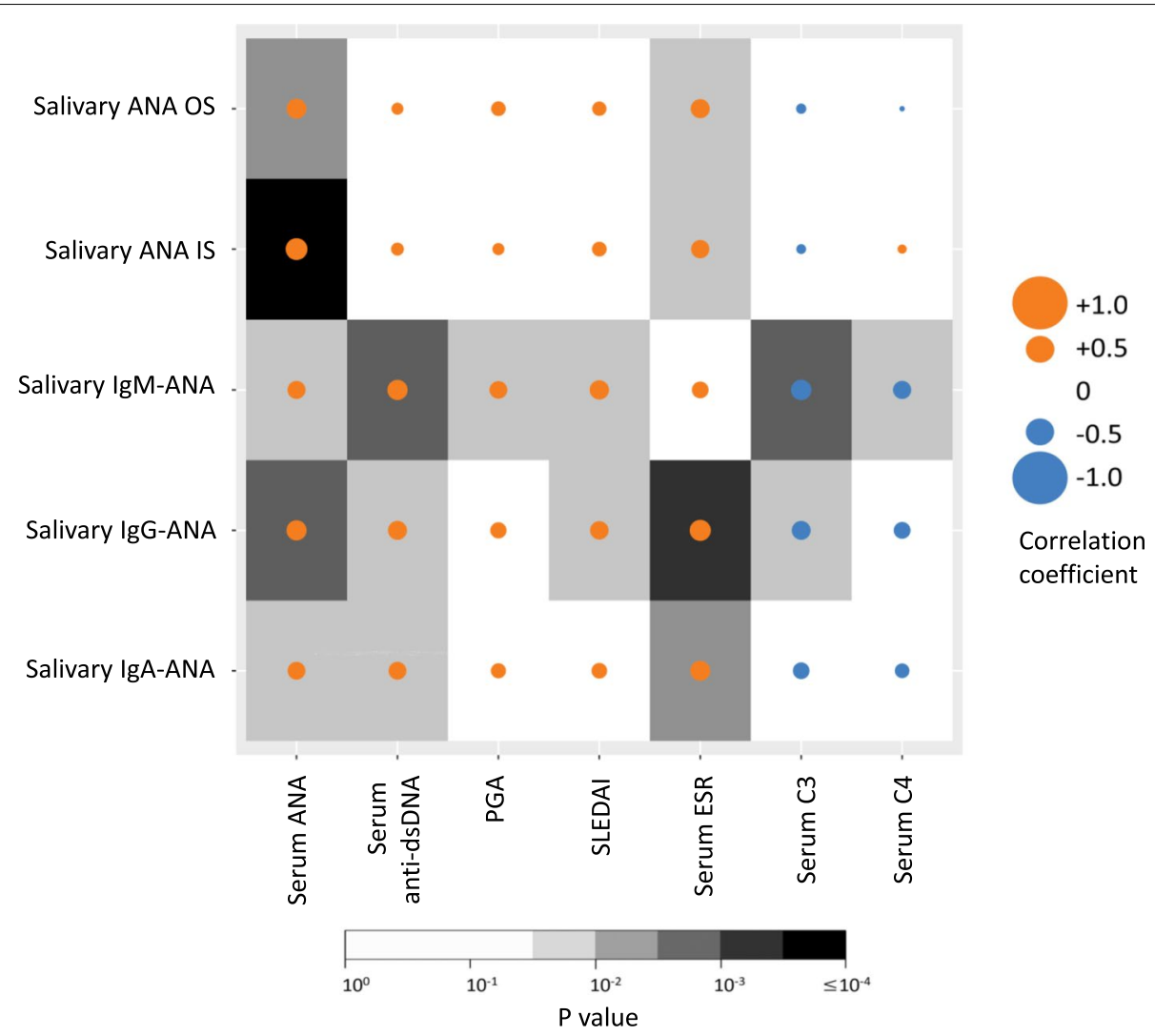

Fig. 4 Correlation of salivary ANA and ANA isotypes with clinical parameters in SLE patients. Salivary ANA OS represents the average score from four observers. Both salivary ANA OS and IS indicate the immunofluorescent intensity of salivary ANA, whereas salivary IgM-ANA, IgG-ANA, and IgA-ANA reflect the ELISA-assayed concentrations of the ANA isotypes. The number of SLE patients assayed was 70. Analysis was done using Spearman correlation. Yellow and blue circles represent positive and negative correlation coefficient, respectively, with larger circles indicating stronger correlation. ESR, erythrocyte sedimentation rate; IS, Image J score; OS, observer score; PGA, physician global assessment; SLEDAl, systemic lupus erythematosus disease activity index

result from oral lesions because only one patient in our study exhibited mucosal ulcers.

The functions of different ANA isotypes also remain unclear. Few studies have investigated ANA isotypes even in the context of serum. Neither IgM-ANA nor IgG-ANA was found to be specific to any particular connective tissue disease, given that they are also detected in rheumatoid arthritis, scleroderma, and other rheumatic diseases. Serum IgG-ANA and IgM-ANA, IgG-ANA alone, and IgM-ANA alone occurred in $59.25 \%, 24.5 \%$, and $4.1 \%$ of SLE patients, respectively [36]. In one study, serum IgGANA, IgM-ANA, and IgA-ANA were measured in SLE patients, discoid lupus erythematosus (DLE) patients, and controls with non-autoimmune diseases. IgM-ANA and IgG-ANA concentrations were significantly elevated in SLE patients compared to DLE patients and controls, and the IgA-ANA concentration was higher in SLE and DLE than in controls. However, the correlations between these isotypes and clinical or laboratory parameters were not evaluated [8]. IgM-ANA failed to produce LE cells as IgG-ANA did [9]. Whereas some IgG autoantibodies are pathogenic, IgM autoantibodies are associated with a wide spectrum of effects, ranging from injurious to protective effects depending on cellular and molecular context [37-40]. Assays using multiplexed proteome microarrays identified two IgG reactivity clusters associated with disease activity and an IgM polyreactive cluster associated with reduced disease activity in the sera of lupus patients [41].

To our knowledge, this study is the first to investigate salivary ANA and ANA isotypes in SLE patients. The IF intensity of salivary ANA and the concentration of each salivary ANA isotype significantly correlated with serum ANA levels. This result highlights the potential of using salivary ANA as a reflection of serum ANA titers, particularly in point-of-care scenarios. Interestingly, the levels of salivary ANA isotypes correlated with several clinical and conventional parameters reflective of disease activity, including SLEDAI, PGA, anti-dsDNA antibody, ESR and $\mathrm{C} 3 / \mathrm{C} 4$ with the latter being negatively 


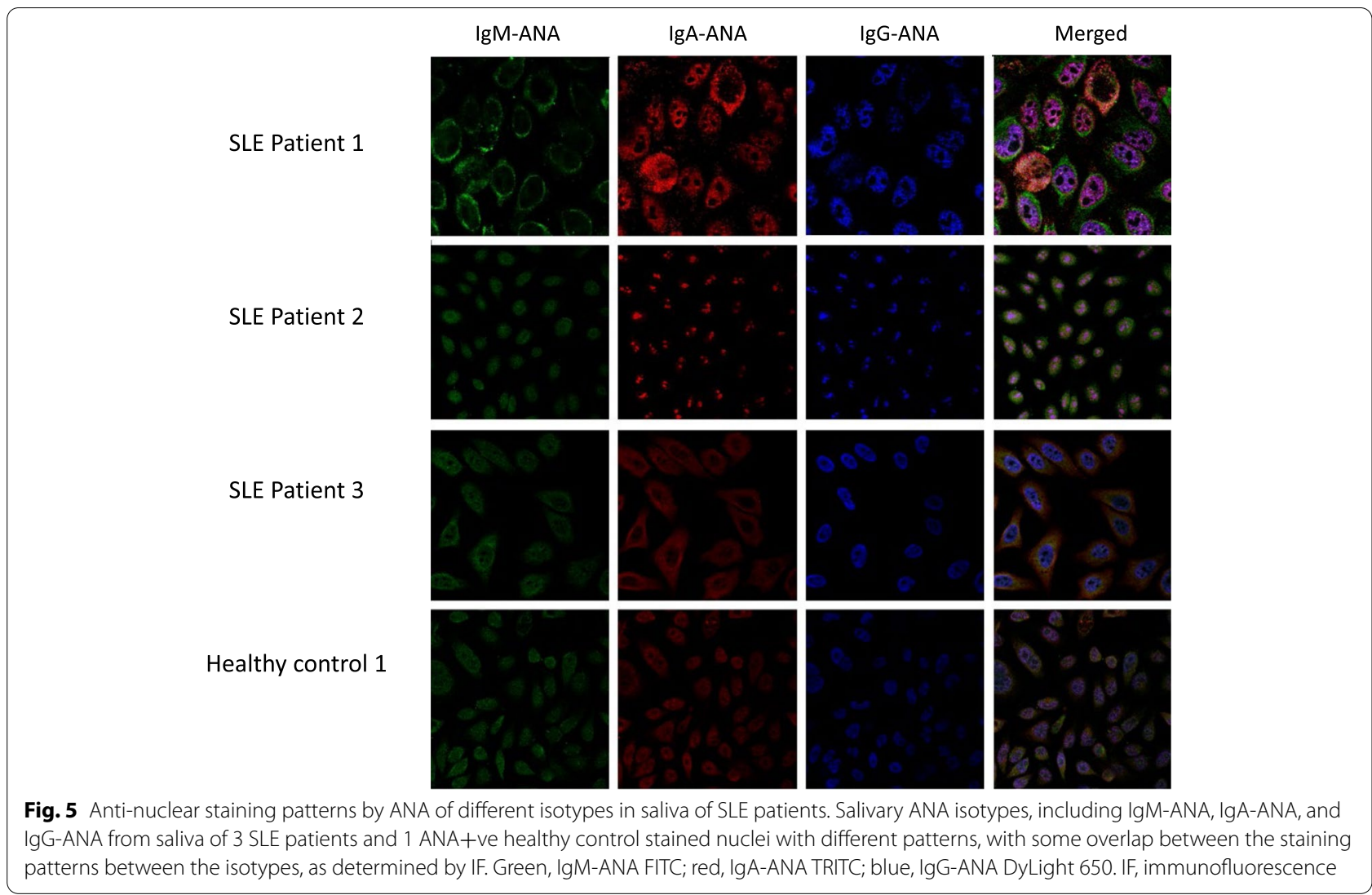

associated. Although serum ANA levels have not been considered useful serial markers of change in disease activity [42], serum ANA titers may be relevant to a patient's immunological profile, and a positive value may signify greater disease activity [43]. Also of interest is the observation that IgA ANAs exhibited $80 \%$ sensitivity for identifying SLE, underscoring its potential utility in screening applications, particularly if this is validated in additional cohorts.

In our study, salivary ANA was detected by IF in $10 \%$ of healthy controls and $67.14 \%$ of SLE patients at the salivary ANA OS cutoff value of 0.75 . Although the SLE classification criteria include ANA, as many as $20 \%$ of otherwise healthy individuals are ANA positive depending on the assay and the titer used as the cutoff for HEp2IF [42].

ANA negativity also occurs in established SLE patients. In a cohort of 1137 SLE patients, 6.2\% were ANA negative by serum IF [44]. Sera from a cohort of 103 SLE patients with historically positive ANA were assayed by three different IF kits, and the frequency of ANA negativity varied from 4.9 to $22.3 \%$ [45]. Similarly, in a cohort of 181 SLE patients with historically positive ANA and clinically active disease, five different IF kits indicated ANA negativity rates from 0.6 to $27.6 \%$ [43]. Variability in ANA negativity exists, especially in individuals with lower ANA titer [46]. In the present study, the ANA negativity in saliva may be attributed to assay and kit variability and the low abundance of salivary ANA, which restricted us from serially diluting saliva samples to determine the ANA isotype titers as regularly performed with serum samples.

One limitation of this study is the limited number of healthy control samples. Inclusion of saliva samples from control patients with other diseases, including aphthous ulcer or Behcet's disease, and other ANApositive autoimmune diseases, may provide additional insights. Whether the composition of salivary ANA isotypes differs between unstimulated and stimulated whole saliva is unknown. Because ANA prevalence is modestly higher in African Americans than in whites [3], validation in a larger cohort of SLE patients from multiple ethnicities is needed. The IF studies to establish the staining patterns of nuclear/cytoplasmic signals also needs to be repeated with further dilutions of the saliva, as recommended [47]. Finally, given the potential functional differences of ANA isotypes, further mechanistic studies are also warranted. 


\section{Conclusion}

Since saliva samples are easy to obtain, the main utility of salivary ANA might be in point-of-care testing and screening, although more evidence and investigations are needed before it could be applied in clinical settings. Given that salivary ANA correlated with serum ANA titer, ANA isotypes correlated with several SLE disease activity indicators, and IgA ANA had high sensitivity for SLE, salivary ANA testing warrants careful and systematic evaluation of its diagnostic and disease monitoring potential. If validated, this could pave the way towards saliva-based point-of-care ANA tests that could be performed even in a primary care setting, for rapid diagnostic evaluation of rheumatic autoimmune diseases.

\begin{abstract}
Abbreviations
ANA: Anti-nuclear antibody; ELISA: Enzyme-linked immunosorbent assay; ESR: Erythrocyte sedimentation rate; FITC: Fluorescein isothiocyanate; HEp-2: Human epithelial type-2; HRP: Horseradish peroxidase; IF: Immunofluorescence; Ig: Immunoglobulin; IS: ImageJ score; OS: Observer score; PBS: Phosphate-buffered saline; PGA: Physician global assessment; pSS: Primary Sjögren's syndrome; SLE: Systemic lupus erythematosus; SLEDAI: SLE disease activity index; rSLEDAI: Renal domains of SLEDAI.
\end{abstract}

\section{Supplementary Information}

The online version contains supplementary material available at https://doi. org/10.1186/s13075-021-02694-6.

Additional file 1: Supplementary Table S1. Characteristics of SLE patients and healthy controls. Supplementary Table S2. Correlation of salivary ANA and ANA isotypes with clinical parameters. Supplementary Figure S1. ROC analysis indicating the discriminatory potential of combined ANA isotypes in discriminating SLE $(n=70)$ from $H C(n=10)$. Sen., sensitivity; Spec., specificity; $A \cup C=$ Area under $(R O C)$ curve.

\section{Acknowledgements}

We thank all participants and patients for this study.

\section{Authors' contributions}

CM conceptualized and designed the study. DG and MP collected the samples and clinical information. TZ, YD, QW, HL, and TN performed the experiments. TZ, GG, and VD did the data analysis. TZ and CM drafted the manuscript. All authors read and approved the final manuscript.

\section{Funding}

This work was supported by NIH grants R21-AR-072911, R01-AR-043727, and R01-AR-069572.

\section{Availability of data and materials}

The data underlying this article will be shared on reasonable request to the corresponding author.

\section{Declarations}

Ethics approval and consent to participate

Informed consent was obtained from each participant, and the study was approved by the Institutional Review Boards of the JHU School of Medicine and the University of Houston.

\section{Consent for publication}

Not applicable.

\section{Competing interests}

The authors declare that they have no competing interests.

\section{Author details}

${ }^{1}$ Biomedical Engineering Department, University of Houston, 3517 Cullen Blvd, Room 2027, TX, Houston, USA. ${ }^{2}$ Present affiliation: The Second Affiliated Hospital, Zhejiang University School of Medicine, Hangzhou, China. ${ }^{3}$ Division of Rheumatology, Johns Hopkins University School of Medicine, Baltimore, MD, USA.

Received: 24 February 2021 Accepted: 7 December 2021

Published online: 03 January 2022

\section{References}

1. Pisetsky DS, Rovin BH, Lipsky PE. New perspectives in rheumatology: biomarkers as entry criteria for clinical trials of new therapies for systemic lupus erythematosus: the example of antinuclear antibodies and antiDNA. Arthritis Rheum. 2017;69(3):487-93.

2. Aringer M, Costenbader K, Daikh D, Brinks R, Mosca M, Ramsey-Goldman $\mathrm{R}$, et al. 2019 European league against rheumatism/American College of Rheumatology classification criteria for systemic lupus erythematosus. Ann Rheum Dis. 2019;78(9):1151-9.

3. Satoh M, Chan EK, Ho LA, Rose KM, Parks CG, Cohn RD, et al. Prevalence and sociodemographic correlates of antinuclear antibodies in the United States. Arthritis Rheum. 2012;64(7):2319-27.

4. Pisetsky DS. Antinuclear antibody testing - misunderstood or misbegotten? Nat Rev Rheumatol. 2017;13(8):495-502.

5. Petri M, Orbai AM, Alarcon GS, Gordon C, Merrill JT, Fortin PR, et al. Derivation and validation of the systemic lupus international collaborating clinics classification criteria for systemic lupus erythematosus. Arthritis Rheum. 2012;64(8):2677-86.

6. Tsokos GC, Lo MS, Costa Reis P, Sullivan KE. New insights into the immunopathogenesis of systemic lupus erythematosus. Nat Rev Rheumatol. 2016;12(12):716-30.

7. Mohan C, Putterman C. Genetics and pathogenesis of systemic lupus erythematosus and lupus nephritis. Nat Rev Nephrol. 2015;11(6):329-41.

8. Jost SA, Tseng LC, Matthews LA, Vasquez R, Zhang S, Yancey KB, et al. IgG, IgM, and IgA antinuclear antibodies in discoid and systemic lupus erythematosus patients. ScientificWorldJournal. 2014;2014:171028.

9. Blondin C, McDuffie FC. Role of lgG and lgM antinuclear antibodies in formation of lupus erythematosus cells and extracellular material. Arthritis Rheum. 1970;13(6):786-97.

10. Methodology of Testing Antinuclear Antibodies Position Statement. ACR:https://www.rheumatology.org/Portals/0/Files/Methodology\% 20of\%Testing\%Antinuclear\%Antibodies\%Position\%Statement.pdf. Accessed 10 Dec 2018.

11. Copple SS, Sawitzke AD, Wilson AM, Tebo AE, Hill HR. Enzyme-linked immunosorbent assay screening then indirect immunofluorescence confirmation of antinuclear antibodies: a statistical analysis. Am J Clin Pathol. 2011;135(5):678-84

12. Choi BY, Yoon MJ, Shin K, Lee YJ, Song YW. Characteristics of pleural effusions in systemic lupus erythematosus: differential diagnosis of lupus pleuritis. Lupus. 2015;24(3):321-6.

13. Hirohata S, Sakuma Y, Yanagida T, Yoshio T. Association of cerebrospinal fluid anti-Sm antibodies with acute confusional state in systemic lupus erythematosus. Arthritis Res Ther. 2014;16(5):450.

14. Pollard KMFL, Webb J. Anti-Sm and anti-DNA antibodies in paired serum and synovial fluid samples from patients with SLE. Rheumatol Int. 1988;8(5):197-204.

15. Meryhew NL, Messner RP, Tan EM. Urinary excretion of antinuclear antibodies. J Rheumatol. 1983;10(6):913-9.

16. Chiappin S, Antonelli G, Gatti R, De Palo EF. Saliva specimen: a new laboratory tool for diagnostic and basic investigation. Clin Chim Acta. 2007:383(1-2):30-40.

17. Van Nieuw AA, Bolscher JG, Veerman EC. Salivary proteins: protective and diagnostic value in cariology? Caries Res. 2004;38(3):247-53.

18. Lorenzo-Pouso Al, Perez-Sayans M, Bravo SB, Lopez-Jornet P, Garcia-Vence $M$, Alonso-Sampedro $M$, et al. Protein-based salivary profiles as novel biomarkers for oral diseases. Dis Markers. 2018;2018:6141845. 
19. Rao PV, Reddy AP, Lu X, Dasari S, Krishnaprasad A, Biggs E, et al. Proteomic identification of salivary biomarkers of type-2 diabetes. J Proteome Res. 2009:8(1):239-45.

20. Yeh CKCN, Floriano PN, Miller CS, Ebersole JL, Weigum SE, McDevitt J, et al. Current development of saliva oral fluid-based diagnostics. Tex Dent J. 2010;127(7):651-61

21. Xiao H, Zhang L, Zhou H, Lee JM, Garon EB, Wong DT. Proteomic analysis of human saliva from lung cancer patients using two-dimensional difference gel electrophoresis and mass spectrometry. Mol Cell Proteomics. 2012;11(2):M111 012112.

22. Wei P, Li C, Qiang L, He J, Li Z, Hua H. Role of salivary anti-SSA/B antibodies for diagnosing primary Sjogren's syndrome. Med Oral Patol Oral Cir Bucal. 2015:20(2):e156-60.

23. Halse AKMM, Wahren-Herlenius M, Jonsson R. Isotype distribution of antiRo SS-A and anti-La SS-B antibodies in plasma and saliva of patients with Sjögren's syndrome. Scand J Rheumatol. 2000;29(1):13-9.

24. Ben-Chetrit EFR, Rubinow A. Anti-SSA Ro and anti-SSB La antibodies in serum and saliva of patients with Sjogren's syndrome. Clin Rheumatol. 1993;12(4):471-4.

25. Chan EK, Damoiseaux J, Carballo OG, Conrad K, de Melo CW, Francescantonio PL, et al. Report of the first international consensus on standardized nomenclature of antinuclear antibody HEp-2 cell patterns 2014-2015. Front Immunol. 2015;6:412.

26. Amado FM, Vitorino RM, Domingues PM, Lobo MJ, Duarte JA. Analysis of the human saliva proteome. Expert Rev Proteomics. 2005;2(4):521-39.

27. Zhao M, Yang Y, Guo Z, Shao C, Sun H, Zhang Y, et al. A comparative proteomics analysis of five body fluids: plasma, urine, cerebrospinal fluid, amniotic fluid, and saliva. Proteomics Clin Appl. 2018;12(6):e1800008.

28. Yan W, Apweiler R, Balgley BM, Boontheung P, Bundy JL, Cargile BJ, et al. Systematic comparison of the human saliva and plasma proteomes. Proteomics Clin Appl. 2009;3(1):116-34.

29. Dawes C, Pedersen AM, Villa A, Ekstrom J, Proctor GB, Vissink A, et al. The functions of human saliva: a review sponsored by the world workshop on Oral medicine VI. Arch Oral Biol. 2015;60(6):863-74.

30. Grassl N, Kulak NA, Pichler G, Geyer PE, Jung J, Schubert S, et al. Ultradeep and quantitative saliva proteome reveals dynamics of the oral microbiome. Genome Med. 2016;8(1):44.

31. L-vKP C-BG, Weterman IT, Biemond I, Peña AS. Immunological findings in whole and parotid saliva of patients with Crohn's disease and healthy controls. Dig Dis Sci. 1984:29(12):1089-92.

32. Ben-Aryeh HGN, Szargel R, Toubi E, Laufer D. Whole saliva in systemic lupus erythematosus patients. Oral Surg Oral Med Oral Pathol. 1993;75(6):696-9.

33. Giuca MR, Pasini M, Tecco S, Giuca G, Marzo G. Levels of salivary immunoglobulins and periodontal evaluation in smoking patients. BMC Immunol. 2014;15:5.

34. Brandtzaeg P. Do salivary antibodies reliably reflect both mucosal and systemic immunity? Ann N Y Acad Sci. 2007;1098:288-311.

35. Grundbacher FJ. Variation in levels of immunoglobulins $\mathrm{a}, \mathrm{G}$ and $\mathrm{E}$ in human saliva. Arch Oral Biol. 1988;33(2):121-6.

36. Chellingworth MC, Salmon M, Scott DL, Bacon PA. The significance of IgM antinuclear antinuclear antibody in rheumatoid arthritis and other connective tissue diseases. Rheumatol Int. 1984;4(1):23-5.

37. Silverman GJ, Vas J, Gronwall C. Protective autoantibodies in the rheumatic diseases: lessons for therapy. Nat Rev Rheumatol. 2013;9(5):291-300.

38. Fereidan-Esfahani M, Nayfeh T, Warrington A, Howe CL, Rodriguez M. IgM natural autoantibodies in physiology and the treatment of disease. Methods Mol Biol. 2019;1904:53-81.

39. Ehrenstein MR, Notley CA. The importance of natural lgM: scavenger, protector and regulator. Nat Rev Immunol. 2010;10(11):778-86.

40. Villalta D, Bizzaro N, Bassi N, Zen M, Gatto M, Ghirardello A, et al. AntidsDNA antibody isotypes in systemic lupus erythematosus: IgA in addition to lgG anti-dsDNA help to identify glomerulonephritis and active disease. PLoS One. 2013;8(8):e71458.

41. Li QZ, Xie C, Wu T, Mackay M, Aranow C, Putterman C, et al. Identification of autoantibody clusters that best predict lupus disease activity using glomerular proteome arrays. J Clin Invest. 2005;115(12):3428-39.

42. Pisetsky DS, Bossuyt X, Meroni PL. ANA as an entry criterion for the classification of SLE. Autoimmun Rev. 2019;18(12):102400.
43. Pisetsky DS, Thompson DK, Wajdula J, Diehl A, Sridharan S. Variability in antinuclear antibody testing to assess patient eligibility for clinical trials of novel treatments for systemic lupus erythematosus. Arthritis Rheum. 2019;71(9):1534-8.

44. Choi MY, Clarke AE, St Pierre Y, Hanly JG, Urowitz MB, Romero-Diaz J, et al. Antinuclear antibody-negative systemic lupus erythematosus in an international inception cohort. Arthritis Care Res. 2019;71(7):893-902.

45. Pisetsky DS, Spencer DM, Lipsky PE, Rovin BH. Assay variation in the detection of antinuclear antibodies in the sera of patients with established SLE. Ann Rheum Dis. 2018;77(6):911-3.

46. Chevrier MJJ, Schreiter J, Benson J. Comparative analysis of anti-nuclear antibody testing using blinded replicate samples reveals variability between commercial testing laboratories [abstract]. Arthritis Rheum. 2016;68(suppl 10): abstract number 2809.

47. Sack U, Conrad K, Csernok E, Frank I, Hiepe F, Krieger T, et al. Autoantibody detection using indirect immunofluorescence on HEp-2 cells. Ann N Y Acad Sci. 2009;1173:166-73.

\section{Publisher's Note}

Springer Nature remains neutral with regard to jurisdictional claims in published maps and institutional affiliations.
Ready to submit your research? Choose BMC and benefit from:

- fast, convenient online submission

- thorough peer review by experienced researchers in your field

- rapid publication on acceptance

- support for research data, including large and complex data types

- gold Open Access which fosters wider collaboration and increased citations

- maximum visibility for your research: over $100 \mathrm{M}$ website views per year

At BMC, research is always in progress.

Learn more biomedcentral.com/submissions 